\title{
RIESGO SEXUAL EN ADOLESCENTES SEGÚN LA EDAD DE DEBUT SEXUAL ${ }^{1}$
}

\author{
JOSÉ P. ESPADA*, ALEXANDRA MORALES, MIREIA ORGILÉS \\ UNIVERSIDAD MIGUEL HERNÁNDEZ - ESPAÑA
}

Recibido, marzo 19/2013

Concepto evaluación, diciembre 12/2013

Aceptado, diciembre 17/2013
Referencia: Espada, J.P., Morales, A. \& Orgilés, M. (2014). Riesgo sexual en adolescentes según la edad de debut sexual. Acta Colombiana de Psicología, 17(1), 53-60. doi: 10.14718/ACP.2014.17.1.6

Resumen

En España los jóvenes debutan sexualmente antes que en otros países; sin embargo, poco se sabe de la relación entre la edad de debut sexual y el comportamiento sexual en esta población. Se analizan las prácticas sexuales y el uso del preservativo según la edad de debut sexual en adolescentes españoles sexualmente experimentados $(N=351)$; la edad media fue 15.9 años $(D T=.75$, rango: 14-18). Se establecieron tres grupos a partir de la edad de debut sexual informada: debut sexual temprano (anterior a los 15 años), promedio (15 años) y tardío (posterior a los 15 años). El grupo con debut sexual temprano practicó en mayor medida masturbación mutua, coito vaginal y sexo oral, con un número de parejas sexuales superior al resto. El uso del preservativo en la primera relación sexual, el porcentaje de uso y el uso consistente del preservativo fue inferior en el grupo debut sexual temprano respecto a los otros dos. El debut sexual anterior a los 15 años se asoció con mayor riesgo de contraer infecciones sexuales por déficit en el uso del preservativo y mayor exposición sexual. Los hallazgos de este estudio sugieren que la edad de debut sexual tiene implicaciones en la salud sexual de los jóvenes.

Palabras clave: Debut sexual, Uso del preservativo, Riesgo sexual, Adolescencia.

\section{SEXUAL RISK IN ADOLESCENTS DEPENDING ON THE AGE OF SEXUAL DEBUT}

\begin{abstract}
In Spain young people have their first sexual intercourse at an earlier age than in other countries; however, little is known about the relationship between age of sexual debut and sexual behavior in this population. Sexual practices and methods of protection are analyzed depending on the age of sexual debut among Spanish adolescents who are sexually experienced $(N=$ $351)$. The mean age was 15.9 years $(S D=.75$, range: $14-18)$. Three groups were formed according to the age of sexual debut reported: early sexual debut (earlier than 15 years), average (15 years) and late (later than 15 years). The early sexual debut group reported further mutual masturbation, vaginal intercourse and oral sex with a higher number of sexual partners than the rest. Condom use at first intercourse, percentage of use and consistent condom use was significantly lower in the early sexual debut group compared to the others. Sexual debut before 15 years old was associated with increased risk of sexual infections, deficit in condom use and increased sexual exposure. The findings of this study suggest that the age of sexual debut has implications for the sexual health in young people.

Key words: Sexual debut, Condom use, Sexual risk, Adolescence.
\end{abstract}

\section{RISCO SEXUAL EM ADOLESCENTES DE ACORDO COM A IDADE DE DEBUTE SEXUAL}

\begin{abstract}
Resumo
$\mathrm{Na}$ Espanha os jovens debutam sexualmente antes que em outros países; porém pouco se sabe com respeito à relação entre idade de debute sexual e o comportamento sexual nesta população. Analisam-se as práticas sexuais e o uso do preservativo
\end{abstract}

\footnotetext{
* Universidad Miguel Hernández, Departamento de Psicología de la Salud. Avda. de la Universidad s/n. 03202 Elche, 11 Departamento (Alicante), España.jpespada@umh.es.

1 Este trabajo ha sido realizado gracias a la Fundación para la Investigación y Prevención del Sida en España (FIPSE) (Ref. 360971/10) y la ayuda concedida a la segunda autora por la Generalitat Valenciana (Programa Vali+D para investigadores en formación) (Ref. $\mathrm{ACIF} / 2012 / 132)$.
} 
segundo a idade de debute sexual em adolescentes espanhóis sexualmente experimentados $(N=351)$; a idade média foi 15.9 anos $(D T=.75$, faixa: 14-18). Estabeleceram-se três grupos a partir da idade de debute sexual informada: debute sexual precoce (anterior aos 15 anos), média (15 anos) e tardio (posterior aos 15 anos). O grupo com debute sexual precoce praticou em maior medida masturbação mútua, coito vaginal e sexo oral, com um número de parceiros sexuais superior ao resto. $\mathrm{O}$ uso do preservativo na primeira relação sexual, a porcentagem de uso e o uso consistente do preservativo foi inferior no grupo de debute sexual precoce com respeito aos outros dois. O debute sexual anterior aos 15 anos foi associado com maior risco de contrair infecções sexuais por déficit no uso do preservativo e maior exposição sexual. Os descobrimentos deste estudo sugerem que a idade de debute sexual tem implicações na saúde sexual dos jovens.

Palavras chave: Debute sexual, Uso do preservativo, Risco sexual, Adolescência.

\section{INTRODUCCIÓN}

Durante la adolescencia y la primera juventud se mantienen las primeras relaciones íntimas y se inicia el periodo de exposición a las infecciones de transmisión sexual (ITS). La edad del primer coito se ha reducido considerablemente en las últimas décadas, situándose antes de los 18 años en muchos países (Wellings et al., 2006). En España, un elevado porcentaje de jóvenes debuta sexualmente alrededor de los 15 años (Espada, Morales, Orgilés, Piqueras y Carballo, 2013; Espada, Orgilés, Morales, Ballester y Huedo-Medina, 2012; Teva, Bermúdez y Buela-Casal, 2009). Diversos estudios señalan la relación entre la edad de debut sexual y el riesgo sexual, en términos de contraer una ITS (Olesen et al., 2011; Schofield, Biernman, Heinrichs y Nix, 2008; Tenkorang y Maticka-Tyndale, 2008) y tener un embarazo no deseado y/o aborto (Cooper et al., 2007; Ma et al., 2009). El debut sexual anterior a los 13 años incrementa dos veces la probabilidad de infectarse por el VIH respecto a quienes tienen la primera experiencia sexual a los 17 años (Kaestle, Halpern, Miller y Ford, 2005). Por consiguiente, el debut sexual temprano (DST) constituye un factor relacionado con el riesgo sexual.

El elevado riesgo de contraer una ITS y/o embarazo no deseado entre los adolescentes con DST podría deberse a un uso deficiente y/o incorrecto de los métodos de protección. Estudios que analizan los métodos de protección en función de la edad de debut sexual encuentran mayor uso del preservativo en jóvenes con inicio sexual tardío (Karibu y Orpinas, 2009; Zimmer-Gembeck y Helfand, 2008). Por el contrario, otros estudios hallan mayor uso del preservativo en los jóvenes que se inician sexualmente temprano respecto a los que el debutan más tarde (Gregson et al., 2002; Hallett, Lewis, et al., 2007). Este resultado puede deberse a la instauración del hábito de uso del preservativo y/o a la mayor conciencia del riesgo en las personas que debutan sexualmente antes que la media de su grupo (Stulhofer, Bacak, Ajdukovic y Graham, 2010).

El DST implica ser sexualmente activo más tiempo y estar expuesto al riesgo antes de quienes se inician después (CDC, 1999). Por consiguiente, el DST se asocia a un his- torial de parejas sexuales más amplio respecto a los que se inician más tardíamente (Dixon-Mueller, 2009; Kaestle et al., 2005; Louie et al., 2009). Los jóvenes que informan de DST hacen menor uso de métodos de protección y presentan mayor riesgo de contraer una ITS con varias parejas (Juárez y LeGrand, 2005; Piña, 2009). Además del riesgo sexual asociado al DST, las intervenciones preventivas ofrecen mejores resultados en jóvenes no activos sexualmente (Espada et al., 2012; Givaudan, Leenen, Van de Vijver, Poortinga y Pick, 2008; UNESCO, 2010). Por este motivo una de las prioridades de las autoridades competentes en prevención del VIH es retrasar la edad de inicio sexual en adolescentes. No obstante, algunos expertos coinciden en que esa medida ayuda al propósito, pero no garantiza una reducción significativa de la tasa de VIH (Hallett, Gregson, et al., 2007; Hallett, Lewis, et al., 2007; Kaestle et al., 2005). Hay evidencia de que el riesgo de contraer una ITS no difiere entre los jóvenes que se inician temprano y los que han estado teniendo sexo a lo largo de su adolescencia (Kaestle et al., 2005). La identificación de comportamientos sexuales que expliquen la relación entre la edad de debut sexual y las ITS permitiría enfocar más acertadamente las intervenciones dirigidas a reducir el riesgo sexual entre los jóvenes.

En España se ha profundizado, por un lado, en el estudio de los factores sociodemográficos y psicosociales que median en el debut sexual (Castro et al., 2011), y por otro lado en los comportamientos sexuales de los adolescentes y jóvenes españoles (Faílde, Lameiras y Bimbela, 2008; Puente et al., 2011). Se sabe sobre la relación entre la edad de debut sexual y otros comportamientos sexuales en mujeres adultas (de Sanjosé et al., 2008). Sin embargo, los adolescentes han sido menos estudiados, a pesar de ser un colectivo con alto riesgo de contraer ITS (Teva, Bermúdez y Buela-Casal, 2011) y del menor sesgo de memoria que entraña la recogida de información sobre sexualidad en esta población. El paso del tiempo puede facilitar el olvido de información y detalles. El tiempo transcurrido entre la primera experiencia sexual y la cumplimentación de la encuesta es más breve cuando se evalúa el comportamiento sexual en adolescentes respecto a otras poblaciones de mayor edad. 
De acuerdo con lo expuesto anteriormente, el presente estudio examina los comportamientos sexuales (prácticas sexuales, frecuencia y número de parejas sexuales) en función de la edad de debut sexual de una muestra representativa de adolescentes españoles. En segundo lugar, se analiza el uso del preservativo (uso del preservativo en la primera relación sexual, porcentaje de uso y uso consistente del preservativo) como determinante del riesgo sexual en función de la edad de debut sexual en la misma muestra.

\section{MÉTODO}

\section{Participantes}

Se reclutó una muestra de 832 adolescentes escolarizados en 15 centros escolares de educación secundaria de la zona del levante, centro y norte de España. Se realizó un muestreo estratificado por regiones: $24.6 \%(n=205)$ de Alicante; $17.8 \%(n=148)$ de Castellón; $16.2 \%(n=135)$ de Madrid; $20.7 \%(n=172)$ de Oviedo, y $20.7 \%(n=172)$ de Murcia. El nivel socioeconómico predominante fue la clase media (60\%), aunque el 32\% era de clase baja, de acuerdo con la escala de Boyce, Torsheim, Currie y Zambon (2006). Del total, fueron seleccionados los que informaron haber debutado sexualmente $(N=351)$, que representan el $42 \%$ de la muestra. La edad media de este grupo fue 15.9 años $(D T=.74$, rango: $14-18)$, y el $54 \%$ fueron chicas.

\section{Variables e instrumento}

Se administró un cuestionario sobre hábitos sexuales (elaborado ad hoc) con base en procedimientos convencionales (Aspy et al., 2007; Bersamin et al., 2008). Para seleccionar a los participantes de este estudio se les preguntó por: a) actividad sexual (“ ¿Alguna vez has tenido una relación sexual con penetración?”) con respuesta dicotómica (Sí/No). Se indagó sobre los comportamientos sexuales y métodos de protección de los estudiantes sexualmente activos: b) edad de la primera relación sexual ("¿Qué edad tenías en el momento de tu primera relación sexual con penetración?"); c) tipo de relaciones sexuales (“¿Qué tipo de prácticas sexuales has mantenido en los últimos seis meses?") con respuesta múltiple (masturbación mutua, sexo vaginal, sexo anal y sexo oral); d) número de parejas sexuales ("¿Con cuántas personas has mantenido relaciones sexuales con penetración en los últimos seis meses?") y e) frecuencia de las relaciones sexuales (" ¿Con qué frecuencia has mantenido masturbación mutua, sexo vaginal, anal $\mathrm{u}$ oral en los últimos seis meses?").

Se incluyeron dos preguntas sobre el preservativo: f) uso del preservativo en la primera relación sexual con penetración ("¿Empleaste preservativo en tu primera relación sexual con penetración?”), con respuesta dicotómica y; g) uso del preservativo en las posteriores relaciones sexuales con penetración ("¿Qué porcentaje de veces utilizas el preservativo en tus relaciones sexuales con penetración?") con una escala de porcentajes que oscilaba entre $0 \%$ y $100 \%$ de las veces.

\section{Procedimiento}

El estudio fue revisado y aprobado por el comité de ética de la Universidad Miguel Hernández de Elche, en España. Se solicitó permiso a los centros educativos y se obtuvo el consentimiento informado de los participantes y sus padres. La evaluación se realizó en el aula y tuvo una duración aproximada de una hora. Se administraron los autoinformes, se leyeron las instrucciones y se resolvieron las dudas. Los profesores no estuvieron presentes durante la cumplimentación del cuestionario. Se asignaron códigos a los participantes para reducir el grado de deseabilidad social. Los adolescentes no recibieron ningún tipo de refuerzo material por su participación.

\section{Análisis estadísticos}

Se analizaron los datos con el paquete estadístico SPSS 19. En este estudio la variable edad de debut sexual fue analizada como variable categórica en lugar de continua, de acuerdo con Espada, Morales y Orgilés (2013). La media en la edad de inicio sexual como valor de comparación entre grupos ha sido cuestionada porque los valores extremos pueden ofrecer una visión distorsionada de la realidad (véase de Irala, Osorio, Carlos, Ruiz-Canela y López-del Burgo, 2011). Los análisis descriptivos (media, mediana y moda) revelaron que la mayoría de los participantes de este estudio tuvo su primera relación con penetración a los 15 años, coincidiendo con datos previos de muestra adolescente española (Castro et al., 2011; Espada et al., 2012; Espada, Morales, Orgilés, Piqueras, et al., 2013; Teva et al., 2009). Con base en esto, se tomaron los 15 años como valor central y se establecieron tres grupos de acuerdo con la edad de inicio sexual:

1. Anterior a los 15 años (debut temprano),

2. A los 15 años (debut promedio) y

3. Posterior a los 15 años (debut tardío).

Se analizaron las diferencias entre los tres grupos mediante tablas de contingencias (variables categóricas) y ANOVA (variables cuantitativas). Las diferencias entre grupos por pares fueron detectadas mediante la prueba posthoc de Scheffé para comparaciones múltiples. Para obtener la medida de la magnitud de las diferencias, se calcularon los índices de Cohen (1988) y las odds ratio (OR). 


\section{RESULTADOS}

\section{Características sociodemográficas}

La muestra final estuvo compuesta por 351 adolescentes sexualmente experimentados, asignados a tres grupos en función de la edad de debut sexual informada. La edad media de debut sexual fue 15.06 (DT = .77). No hubo diferencias en género, rendimiento académico, ni en situación familiar entre los tres grupos.

Comportamiento sexual: prácticas sexuales, frecuencia y parejas sexuales

Como se muestra en la Tabla 1, los participantes con debut sexual anterior a los 15 años constituyen el grupo que en mayor proporción practicó masturbación mutua, coito vaginal y sexo oral en los seis meses previos a la evaluación. La edad de debut sexual no estuvo relacionada con haber mantenido sexo anal en los últimos seis meses. La frecuencia con la que se implican en relaciones sexuales no difirió estadísticamente entre los tres grupos. Sin embar- go, el número de parejas sexuales durante los seis meses previos a la evaluación fue significativamente superior en los adolescentes con DST respecto al resto $(\mathrm{F}=4.23, \mathrm{p}<$ $.05)$, con un tamaño del efecto medio.

\section{Uso del preservativo}

El preservativo fue el método de protección más empleado, especialmente por quienes se inician más tardíamente. La proporción de adolescentes que utilizaron el preservativo en la primera relación coital fue significativamente inferior en el grupo DST respecto a los grupos con debut promedio y tardío $\left(\mathrm{X}^{2}=11.27 ; p<.01\right)$. El porcentaje de uso del preservativo en las posteriores relaciones sexuales difirió en función de la edad de debut sexual $\left(\mathrm{X}^{2}=3.01\right.$; $p<.05)$. Los grupos con debut promedio y tardío utilizaron este método de protección en el $92 \%$ y $94 \%$ de las veces, mientras que el grupo con debut temprano lo hizo únicamente en el $87 \%$ de las ocasiones. En lo que respecta al uso consistente del preservativo, la tasa fue superior en los adolescentes que debutaron sexualmente después de los 15

Tabla 1

Prácticas sexuales según la edad de debut sexual en adolescentes españoles $(\mathrm{N}=351)$.

\begin{tabular}{|c|c|c|c|c|c|c|}
\hline & $\begin{array}{c}(1) \\
\text { Debut sexual } \\
\text { temprano } \\
N=66\end{array}$ & $\begin{array}{c}(2) \\
\text { Debut sexual } \\
\text { promedio } \\
N=201\end{array}$ & $\begin{array}{c}(3) \\
\text { Debut sexual } \\
\text { tardío } \\
N=84\end{array}$ & Test $^{\mathrm{a}}$ & Post Hoc & $\begin{array}{c}\mathrm{d} / \mathrm{OR} \\
(95 \% \mathrm{IC})\end{array}$ \\
\hline \multicolumn{7}{|l|}{ Prácticas sexuales $N(\%)$} \\
\hline Masturbación mutua & $36(54.5)$ & $102(50.7)$ & $31(36.9)$ & $5.87^{*}$ & $\begin{array}{l}1>2 \\
1>3\end{array}$ & $\begin{array}{c}1.16 \\
(.66,2-03) \\
2.05 \\
(1.06,3.95)\end{array}$ \\
\hline Coito vaginal & $52(78.8)$ & $106(52.7)$ & $66(78.6)$ & $25.01^{* * *}$ & $\begin{array}{l}1>2 \\
3>2\end{array}$ & $\begin{array}{c}3.32 \\
(1.73,6.38) \\
7.59 \\
(3.92,14.69)\end{array}$ \\
\hline $\begin{array}{l}\text { Coito anal } \\
\text { Sexo oral }\end{array}$ & $\begin{array}{l}8(12.1) \\
36(54.5)\end{array}$ & $\begin{array}{c}11(5.5) \\
67(33.3)\end{array}$ & $\begin{array}{c}5(6) \\
20(23.8)\end{array}$ & $\begin{array}{c}3.58 \\
15.94^{* * *}\end{array}$ & $\begin{array}{l}1>2 \\
2>3\end{array}$ & $\begin{array}{c}2.40 \\
10.86 \\
(5.56,21.20)\end{array}$ \\
\hline \multicolumn{7}{|l|}{ Frecuencia $N(\%)$} \\
\hline $1 \mathrm{vez} / \mathrm{mes}$ & $9(17)$ & $45(27.6)$ & $26(36.6)$ & 12.72 & & \\
\hline $2-3$ veces $/$ mes & $15(28.3)$ & $52(31.9)$ & $18(25.4)$ & & & \\
\hline $1-2$ veces/semana & $15(28.3)$ & $42(25.8)$ & $19(26.8)$ & & & \\
\hline 3-4 veces/semana & $9(17)$ & $16(9.8)$ & $5(7)$ & & & \\
\hline $5-7$ veces/semana & $5(9.4)$ & $8(4.9)$ & $3(4.2)$ & & & \\
\hline $\begin{array}{l}\text { Número de parejas sexuales } \\
M(D T)\end{array}$ & $1.93(1.60)$ & $1.14(1.26)$ & $1.30(.95)$ & $4.23^{*}$ & $\begin{array}{l}1>2 \\
1>3\end{array}$ & $\begin{array}{l}.54 \\
.69\end{array}$ \\
\hline
\end{tabular}

Nota. ${ }^{a}$ Estadístico $F$ para variables continuas y $X^{2}$ para variables categóricas. $d=$ índice de Cohen. $O R=$ Odds Ratio. ${ }^{*} p<.05,{ }^{* * *} p<.001$. 
Tabla 2

Uso del preservativo según la edad de debut sexual en adolescentes españoles $(\mathrm{N}=351)$.

\begin{tabular}{|c|c|c|c|c|c|c|}
\hline & $\begin{array}{c}(1) \\
\text { Debut sexual } \\
\text { temprano } \\
N=66\end{array}$ & $\begin{array}{c}(2) \\
\text { Debut sexual } \\
\text { promedio } \\
N=201\end{array}$ & $\begin{array}{c}(3) \\
\text { Debut sexual } \\
\text { tardío } \\
N=84 \\
\end{array}$ & Test $^{\mathrm{a}}$ & Post Hoc & $\begin{array}{c}\mathrm{d} / \mathrm{OR} \\
(95 \% \mathrm{IC}) \\
\end{array}$ \\
\hline \multirow{2}{*}{$\begin{array}{l}\text { Uso del preservativo en la } \\
\text { primera relación } N(\%)\end{array}$} & $51(77.3)$ & $181(90)$ & 79 (94) & $11.27^{* *}$ & $1>2$ & $.37(.17, .78)$ \\
\hline & & & & & $1>3$ & $5.25(2.76,9.97$ \\
\hline \multirow{2}{*}{$\begin{array}{l}\text { Uso consistente preservativo } \\
N(\%)\end{array}$} & $25(47.2)$ & $66(52.4)$ & $48(72.7)$ & $9.82^{* *}$ & $1>3$ & $1.94(1.07,3.51)$ \\
\hline & & & & & $2>3$ & $.36(.21, .61)$ \\
\hline $\begin{array}{l}\% \text { Uso del preservativo } \\
M(D T)\end{array}$ & $87.13(21.9)$ & $91.94(13.18)$ & $93.79(12.02)$ & $3.01^{*}$ & $1>3$ & .38 \\
\hline
\end{tabular}

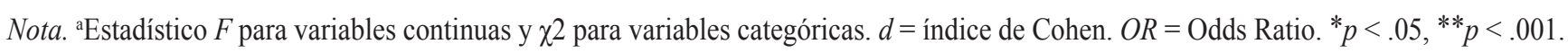

años $(\mathrm{X} 2=9.82 ; \mathrm{p}<.01)$. El $73 \%$ de los que se iniciaron más tardíamente empleó el preservativo consistentemente en sus relaciones sexuales con penetración. Sin embargo, menos de la mitad de los que debutaron antes de los 15 años hizo uso de este método de protección el 100\% de las veces. Los resultados sugieren que el uso del preservativo desciende significativamente a medida que la edad de inicio sexual es más temprana (Tabla 2).

\section{DISCUSIÓN}

El objetivo de este estudio fue analizar la relación de la edad de debut sexual en los comportamientos sexuales y el uso del preservativo en una muestra de adolescentes españoles. Se consideró DST a la primera relación sexual con penetración anterior a los 15 años, coincidiendo con la edad media de debut sexual de los participantes y con estudios anteriores (Olesen et al., 2011; Pettifor, Van der Straten, Dubar, Shiboski y Padian, 2004; Potdar y Mmari, 2011). En España la edad de debut sexual se sitúa en torno a los 15 años (Castro et al., 2011; Teva et al., 2009), unos años por debajo respecto a muestras con cohortes de mayor edad (de Sanjosé et al., 2008; Wellings et al., 2006) y estudios internacionales (Hallett, Lewis, et al., 2007; Haydon, Herring, Prinstein y Halpern, 2011).

El DST se relacionó con mayor variedad de prácticas sexuales (masturbación mutua, coito vaginal y sexo oral) en los últimos seis meses. Aunque no se encontraron diferencias estadísticamente significativas en coito anal, los adolescentes que debutaron sexualmente antes de los 15 años se implicaron en mayor medida en esta práctica sexual, coincidiendo con el estudio de Haydon y colaboradores (2011). Nuestros resultados afianzan la relación entre el
DST y la variedad de prácticas sexuales (Hallett, Lewis, et al., 2007). Como era esperable, los adolescentes con DST informaron de mayor número de parejas sexuales en los últimos seis meses. Este resultado confirma lo señalado por estudios previos (Dixon-Mueller, 2009; Kaestle et al., 2005; Louie et al., 2009; Piña, 2009; Zimmer-Gembeck y Helfand, 2008). Respecto a la relación entre el número de parejas sexuales y el riesgo sexual, los jóvenes que tienen mayor número de parejas sexuales se ajustan a un perfil de uso esporádico del preservativo (Karibu y Orpinas, 2009), lo que conlleva un mayor riesgo sexual para contraer ITS y para que se presenten embarazos no deseados y abortos.

El preservativo es el método de protección sexual de primera elección para los adolescentes. Aun así, se observa que un buen porcentaje no lo utiliza o lo hace puntualmente. El uso del preservativo en la primera relación sexual fue significativamente inferior en los adolescentes que informaron haberse iniciado sexualmente antes de los 15 años. La probabilidad de protegerse de las ITS en las posteriores relaciones sexuales se predice a partir del uso del preservativo en la primera experiencia sexual (Juárez y LeGrand, 2005; Lameiras, Faílde, Bimbela y Alfaro, 2008). De ahí la importancia de promocionar el uso del preservativo antes de que los jóvenes se inicien en los comportamientos sexuales. En lo que respecta a la probabilidad de uso del preservativo en las posteriores relaciones sexuales, estos resultados coinciden con estudios longitudinales (véase Zimmer-Gembeck y Helfand, 2008) en los que se halla mayor uso consistente del preservativo en los adolescentes que se inician más tardíamente, respecto a los que se inician antes. Por lo tanto, las acciones promotoras del uso del preservativo, además de los motivos que subyacen a su uso en los jóvenes (Piña, Lozano, Vázquez y Carrillo, 
2010), deben tener en cuenta la edad de inicio sexual como un factor de riesgo para contraer ITS y para embarazos no deseados o abortos.

El análisis del comportamiento sexual permite identificar diferentes patrones de conducta sexual y uso de métodos de protección en función de la edad de inicio sexual. El debut sexual tardío se relacionó con menor implicación en prácticas sexuales como la masturbación mutua y el sexo oral respecto al DST. Los adolescentes que debutaron sexualmente después de los 15 años informaron de un menor número de parejas sexuales respecto a quienes lo hicieron antes de los 15 años (Media de 1.30 vs. 1.93 parejas sexuales). Este resultado es esperable, dado que los adolescentes con DST tuvieron su primera experiencia antes y han permanecido sexualmente activos durante más tiempo que los que debutaron más tarde. El inicio sexual tardío se asoció con mayor uso del preservativo en la primera experiencia sexual y mayor uso consistente del mismo en las posteriores relaciones sexuales. Mientras que el 72\% de los adolescentes con debut sexual tardío informó de un uso del preservativo en todas sus relaciones, sólo lo hizo el $52 \%$ con debut sexual promedio y el $47 \%$ con DST. Estos hallazgos sugieren que el debut sexual tardío podría ser un factor protector para implicarse en comportamientos sexuales de riesgo, como son el sexo desprotegido y múltiples parejas sexuales.

El debut sexual anterior a los 15 años se relacionó con mayor variedad de prácticas sexuales, mayor número de parejas sexuales y menor uso del preservativo en la primera relación sexual, menor probabilidad de uso del preservativo y menor uso consistente del preservativo respecto a los que se inician más tarde. Estos resultados indican que los jóvenes con DST se ajustan en mayor medida a un perfil comportamental de riesgo sexual por la mayor variedad de actividades sexuales con un mayor número de parejas y el menor uso del preservativo respecto a los que se inician sexualmente más tarde. Se concluye que la edad de inicio sexual se relaciona con el riesgo sexual durante la adolescencia.

El presente estudio tiene una serie de limitaciones a tener en cuenta. La selección de los adolescentes sexualmente activos redujo la muestra considerablemente. En todo caso, las características sexuales de los participantes de este estudio no difieren de las encontradas con extensas muestras de adolescentes españoles (Puente et al., 2011; Faílde et al., 2008). El diseño transversal utilizado no permite establecer relaciones de causalidad entre la edad de debut sexual y los comportamientos sexuales. Sería recomendable llevar a cabo estudios longitudinales para determinar si existe una relación causal. Debido a lo complejo de indagar en los comportamientos sexuales (Catania, Gibson, Chitwood y Coates, 1990), se emplearon medidas de autoinforme anónimas para reducir el efecto de la deseabilidad social.

Este trabajo pone de manifiesto que la edad de debut sexual se relaciona con el comportamiento sexual durante la juventud. El uso del preservativo se redujo considerablemente a medida que la edad de inicio sexual descendió. El debut sexual anterior a los 15 años se relacionó con mayor riesgo de contraer una ITS por déficit en el uso del preservativo. Los hallazgos de este estudio pueden guiar las acciones de prevención y promoción de la salud sexual en población adolescente. Se recomienda que dichas acciones tengan en cuenta la diversidad de perfiles de comportamiento sexual asociados a la edad de debut sexual.

\section{REFERENCIAS}

Aspy, C. B., Vesely, S. K., Oman, R. F., Rodina, S., Marshall, L. D., Fluhr, J. y McLeroy, K. (2007). Parental communication and youth sexual behavior. Journal of Adolescence, 30, 449-466.

Bersamin, M. T., Todd, M., Fisher, D. A., Hill, D. L., Grube, J. W. y Walker, S. (2008). Parenting practices and adolescent sexual behavior: A longitudinal study. Journal of Marriage and Family, 70, 97-112.

Boyce, W., Torsheim, T., Currie, C. y Zambon, A. (2006). The family affluence scale as a measure of national wealth: validation of an adolescent self-report measure. Social Indicators Research, 78(3), 473-487.

Castro, A., Bermúdez, M. P., Buela-Casal, G. y Madrid, J. (2011). Variables psicosociales que median en el debut sexual de adolescentes en España. Revista Latinoamericana de Psicología, 43, 83-94.

Catania, J. A., Gibson, D. R., Chitwood, D. D. y Coates, T. J. (1990). Methodological problems in AIDS behavioral research: Influences on measurement error and participation bias in studies of sexual behavior. Psychological Bulletin, 108, 339-362.

CDC (Centers for Disease Control) (1999). Youth risk behavior surveillance- United States, 1999. Recuperado el 28 de abril de 2012, de http:// www.cdc.gov/MMWR/PDF/ss/ss4905.pdf

Cohen, J. (1988). Statistical power analysis for the behavioral sciences (2nd edition). Hillsdale, NJ: Erlbaum.

Cooper, D., Hoffman, M., Carrara, H., Rosenberg, L., Kelly, J., Stander, I., ...Shapiro, S. (2007). Determinants of sexual activity and its relation to cervical cancer risk among South African women. BMC Public Health, 7, 341. Recuperado el 28 de abril de 2012, de http://www.biomedcentral.com/ content/pdf/1471-2458-7-341.pdf

Dixon-Mueller, R. (2009). Starting Young: Sexual initiation and HIV prevention in early adolescence. AIDS and Behavior, 13, 100-109. 
Espada, J. P., Orgilés, M., Morales, A., Ballester, R. y HuedoMedina, T. (2012). Effectiveness of a School HIV/AIDS Prevention Program for Spanish Adolescents. AIDS Education and Prevention, 24(6), 500-513.

Espada, J. P., Morales, A. y Orgilés, M. (2013). Relación entre la edad de debut sexual y el sexo bajo los efectos de las drogas en la adolescencia. Revista Española de Drogodependencias, 38, 25-35.

Espada, J.P., Morales, A., Orgilés, M., Piquera, J. A. y Carballo, J. L. (2013). Sexual behaviour under the influence of alcohol among Spanish adolescents. Adicciones, 25, 55-62.

Faílde, J. M., Lameiras, M. y Bimbela, J. L. (2008). Prácticas sexuales de chicos y chicas españoles de 14-24 años de edad. Gaceta Sanitaria, 22, 511-519.

Givaudan, M., Leenen, I., Van de Vijver, F. J., Poortinga, Y. H. y Pick, S. (2008). Longitudinal study of a school based HIV/ AIDS early prevention program for Mexican adolescents. Psychology, Health y Medicine, 13, 98-110.

Gregson, S., Nyamukapa, C. A., Garnett, G. P., Mason, P. R., Zhuwau, T., Caraël, M., Anderson, R. M. (2002). Sexual mixing patterns and sex-differentials in teenage exposure to HIV infection in rural Zimbabwe. Lancet, 395, 18961903.

Hallett, T. B., Gregson, S., Lewis, J. J., Lopman, B. A. y Garnett, G. P. (2007). Behaviour change in generalized HIV epidemics: Impact of reducing cross-generational sex and delaying age at sexual debut. Sexual Transmitted Infections, 83, 50-54.

Hallett, T. B., Lewis, J. J. C., Lopman, B. A., Nyamukapa, C. A., Mushati, P., Wamber, M., Gregson, S. (2007). Age at first sex and HIV infection in Rural Zimbabwe. Studies in Family Planning, 38, 1-10.

Haydon, A. A., Herring, A. H., Pristein, M. J. y Halpern, C. T. (2011). Beyond age at first sex: Patterns of Emerging sexual behavior in adolescence and young adulthood. Journal of Adolescence Health, 50(5), 456-463.

de Irala, J.; Osorio, A.; Carlos, S.; Ruiz-Canela, M. y López-del Burgo, C. (2011). Mean age of first sex: Do they know what we mean? Archives of Sexual Behavior, 40, 853-855.

Juárez, F. y LeGrand, T. (2005). Factors influencing boys' age at first intercourse and condom use in the Shantytowns of Recife, Brazil. Studies in Family Planning, 36, 57-70.

Kaestle, C. E., Halpern, C. T., Miller, W. C. y Ford, C. A. (2005). Young age at first sexual intercourse and sexually transmitted infections in adolescents and young adults. American Journal of Epidemiology, 161, 774-780.

Karibu, C. W. y Orpinas, P. (2009). Correlates of condom use among male high school students in Nairobi, Kenya. Journal of School Health, 79, 425-432.

Lameiras, M., Faílde, J. M., Bimbela, J. L. y Alfaro, N. (2008). Uso del preservativo masculino en las relaciones con coito vaginal de jóvenes españoles entre catorce y veinticuatro años. Diversitas: Perspectivas en Psicología, 4, 401-415.
Louie, K. S., de Sanjose, S., Díaz, M., Castellsague, X., Herrero, R., Meijer, C. J., Bosch, F. X. (2009). Early age at first sexual intercourse and early pregnancy are risk factors for cervical cancer in developing countries. British Journal of Cancer, 100, 1191-1197.

Ma, Q., Ono-Kihara, M., Cong, L., Xu, G., Pan, X., Zamani, S., Kihara, M. (2009). Early initiation of sexual activity: A risk factor for sexually transmitted diseases, HIV infection, and unwanted pregnancy among university students in China. BMC Public Health, 9. Recuperado el 30 de abril de 2012, de http://www.ncbi.nlm.nih.gov/pmc/articles/ PMC2674603/pdf/1471-2458-9-111.pdf

Olesen, T. B., Jensen, K. E., Nygard, M., Tryggvadottir, L., Sparen, P., Hansen, B. T., Kjaer, S. K. (2011). Young age at first intercourse and risk-taking- a study of nearly 65000 women in four Nordic countries. The European Journal of Public Health, 90, 459-467.

Pettifor, A. E., Van der Straten, A., Dubar, M. S., Shiboski, S. C. y Padian, N. S. (2004). Early age of first sex: A risk factor for HIV infection among women in Zimbabwe. AIDS, 18, $1435-1442$.

Piña, J. A. (2009). Predictores del comportamiento sexual con múltiples parejas en estudiantes de educación superior: un análisis por género. Acta Colombiana de Psicología, 12, 53-65.

Piña, J. A., Lozano, D. I., Vázquez, P. y Carrillo, I. C. (2010). Motivos y uso de preservativo en estudiantes universitarios de Ciudad Juárez (México). Anales de Psicología, 26, 1826.

Potdar, R. y Mmari, K. (2011). Factors influencing sexual initiation, multiple partners and condom use among male slum youth in Pune, India. Global Public Health, 6, 843-858.

Puente, D., Zabaleta, E., Rodríguez-Blanco, T., Cabanas, M., Monteagudo, M., Pueyo, M. J., Bolíbar, B. (2011). Gender differences in sexual risk behaviour adolescents in Catalonia, Spain. Gaceta Sanitaria, 25, 13-19.

de Sanjosé, S., Cortés, X., Méndez, C., Puig-Tintore, L., Torné, A., Roura, E., Castellsague, X. (2008). Age at sexual initiation and number of sexual partners in the female Spanish population. Results from the AFRODITA survey. European Journal of Obstetrics y Gynecology and Reproductive Biology, 140, 234-240.

Schofield, H. L. T., Biernman, K. L., Heinrichs, B. y Nix, R. L. (2008). Predicting early sexual activity with behavior problems exhibited at school entry and in early adolescence. Journal of Abnormal Child Psychology, 36, 1175-1188.

Stulhofer, A., Bacak, V., Ajdukovic, D. y Graham, C. (2010). Understanding the association between condom use at first sexual intercourse: An assessment of normative, calculative, habitual explanations. Social Science and Medicine, 70, 2080- 2084.

Tenkorang, E. Y. y Maticka-Tyndale, E. (2008). Factors influencing the timing of first sexual intercourse among young people in Nyanza, Kenya. International Family Planning Perspectives, 34, 177-188. 
Teva, I., Bermúdez, M. P. y Buela-Casal, G. (2009). Characteristics of sexual behavior in Spanish adolescents. The Spanish Journal of Psychology, 12, 471-484.

Teva, I., Bermúdez, M. P. y Buela-Casal, G. (2011). Búsqueda de sensaciones sexuales, estilos de afrontamiento, estrés social y su relación con la conducta sexual adolescente. Anales de Psicología, 27, 35-46.

Unesco (2010). Orientaciones técnicas internacionales sobre educación en sexualidad: un enfoque basado en evidencia orientado a escuelas, docentes y educadores de la salud. Re- cuperado el 12 de mayo de 2012, de http://unesdoc.unesco. org/images/0018/001832/183281s.pdf

Wellings, K., Collumbien, M., Slaymaker, E., Singh, S., Hodges, Z., Patel, D. y Bajos, N. (2006). Sexual behaviour in context: A global perspective. Lancet, 368, 1706-1728.

Zimmer-Gembeck, M. J. y Helfand, M. (2008). Ten years of longitudinal research on U.S. adolescent sexual behavior: Developmental correlates of sexual intercourse, and the importance of age, gender and ethnic background. Developmental Review, 28, 153-224. 